\title{
Metabolic syndrome and coronary artery disease in adults with congenital heart disease
}

\author{
Koichiro Niwa \\ Department of Cardiology, Cardiovascular Center, St Luke's International Hospital, Tokyo 104-8560, Japan \\ Correspondence to: Koichiro Niwa, MD, PhD, FACC, FAHA, FJCC. Department of Cardiology, Chiba Kaihin Municipal Hospital, 3-31-1 Isobe, \\ Mihama-ku, Chiba 261-0012 Japan. Email: kniwa@aol.com.
}

\begin{abstract}
In adults with congenital heart disease (ACHD), conditions acquired with aging, such as metabolic syndrome, hypertension, diabetes mellitus, and obesity, can negatively influence the original cardiovascular disease. Metabolic syndrome has a higher prevalence in ACHD than in the general population. In contrast, coronary artery disease shows a similar prevalence in adults with acyanotic CHD and the general population, while adults with cyanotic CHD, even after repair, have an even lower incidence of coronary artery disease than the general population/adults with acyanotic CHD. However, even in those with cyanotic CHD, coronary artery disease can develop when they have risk factors such as obesity, dyslipidemia, hypertension, diabetes mellitus, smoking habit, or limited exercise. The prevalence of risk factors for cardiovascular disease is similar between ACHD and the general population, but an increased risk of coronary atherosclerosis has been observed for congenital coronary artery anomalies, dextro-transposition of the great arteries after arterial switch operation, Ross procedure, and coarctation of the aorta. Aortopathy may be an additional risk factor for cardiovascular disease. As ACHD have other abnormalities that may make the heart more vulnerable to both the development of atherosclerosis and adverse cardiovascular sequelae, regular evaluation of their cardiovascular disease risk status is recommended. Metabolic syndrome is more common among ACHD than in the general population, and may therefore increase the future incidence of atherosclerotic coronary artery disease even in ACHD. Thus, ACHD should be screened for metabolic syndrome to eliminate risk factors for atherosclerotic coronary artery disease.
\end{abstract}

Keywords: Adult congenital heart disease; cardiovascular disease; coronary artery disease; cyanotic congenital heart disease; metabolic syndrome

Submitted Sep 14, 2020. Accepted for publication Oct 27, 2020.

doi: $10.21037 / \mathrm{cdt}-20-781$

View this article at: http://dx.doi.org/10.21037/cdt-20-781

\section{Introduction}

At present, $90 \%$ of patients born with congenital heart disease (CHD) grow to adulthood. Consequently, there are more adults with CHD than children with CHD, and the number is expected to grow (1). Health conditions acquired lesions with aging, such as hypertension, diabetes mellitus, and obesity, can negatively impact the original cardiovascular disease (CVD). Furthermore, atherosclerosis may pose an additional health problem in adults with $\mathrm{CHD}$ (ACHD); ACHD are at risk of developing atherosclerotic CVD when they reach the age at which atherosclerosis becomes clinically relevant (2-10). Empirical evidence suggests that ACHD are also prone to developing coronary artery disease (CAD). The prevalence of cardiovascular risk factors in these patients is similar to that in the general population (6,7). Adults with acyanotic CHD have a similar prevalence of $\mathrm{CAD}$ to the general population $(3,4)$. ACHD may be at increased risk for premature CAD for several reasons: abnormal coronary artery anatomy, inflammation and scarring of the coronary arteries from surgical manipulation or the abnormal anatomy underlying the CHD, reperfusion injuries during cardiac surgery, associated genetic syndromes, ventricular hypertrophy or 
dilation, and aortopathy with dilated aorta and increased aortic stiffness.

ACHD, especially those with cyanotic types of CHD, even after repair, have a minimal incidence of CAD (2,8-12). However, even in those with cyanotic CHD, CAD can develop in those with several risk factors for CAD and limited exercise (4).

Metabolic syndrome is a constellation of risk factors for CVD, including obesity, dyslipidemia, insulin resistance, and hypertension. These risk factors are associated with excessive acquired CVD and type 2 diabetes mellitus, as well as increased mortality in general $(13,14)$. Metabolic syndrome has a higher prevalence in ACHD than in the general population, and its prevalence increases with age $(13,14)$. ACHD are now living longer, with increasing CAD risk, which may increase the incidence of atherosclerotic CAD in ACHD. Atherosclerosis may therefore pose an additional health problem to ACHD as time goes forward (2-6). The development of metabolic syndrome, hypertension, diabetes, dyslipidemia, and obesity also pose significant additional risk in $\operatorname{ACHD}(2,7)$.

\section{Prevalence of CAD in ACHD}

Yalonetsky et al. (4) examined 12,124 ACHD with CAD and reported that $1 \%(141 / 12,124)$ had both CHD and obstructive CAD (age: $56 \pm 13$ years). In these 141 patients, five conditions led to higher prevalence of obstructive CAD: atrial septal defect (35\%), bicuspid aortic valve (18\%), tetralogy of Fallot (TOF) (9\%), coarctation of the aorta (COA) (7\%) and Eisenmenger syndrome $(6 \%)$. Traditional risk factors were commonly observed in these patients $(82 \%)$. In particular, all patients with significant cyanosis and Eisenmenger syndrome had several risk factors for CAD. The researchers concluded that CAD was observed even in adults with cyanotic CHD when they had several traditional risk factors. Systemic arterial hypertension and hyperlipidemia were strong predictors of CAD in these patients. Giamberti et al. (15) reported that, of a total of 1,154 ACHD who underwent surgery, 50 ( $4.3 \%$, mean age 66 years) were diagnosed with acquired CAD and required coronary artery bypass grafting. The primary diagnoses were atrial septal defect $(n=40)$, TOF $(n=4)$, ventricular septal defect $(n=2)$, and others. Stulak et al. performed concomitant coronary artery bypass grafting at the time of CHD repair in 122 patients (median age 64 years) (16). Thirty (25\%) of these patients had preoperative angina, seven $(6 \%)$ had previous myocardial infarction, and six ( $5 \%$ ) had previous percutaneous intervention. The most common primary cardiac diagnoses were secundum atrial septal defect (60\%), Ebstein's anomaly (11\%), and partial anomalous pulmonary venous connection (7\%). Bokma et al. (17) examined 167 patients >50 years of age with repaired TOF, and found during follow-up that 11 had CAD (2 with coronary revascularization and 3 with myocardial infarction; 6 were stable). The incidence of myocardial infarction was similar to that in the general population. Egbe et al. (18) reported that, based on a selected cohort of 105 repaired-TOF patients (mean age $47 \pm 12$ years), the prevalence of mild CAD and significant CAD was $19 \%$ and $15 \%$, respectively. Bokma et al. (19) reported that in 6,904 ACHD, 55 had CAD (mean age $55.1 \pm 12.4$ years) and traditional atherosclerotic risk factors (hypertension, hypercholesterolemia, and smoking). Basuer et al. (20) investigated 539 ACHD and found that $16(3.0 \%)$ had CAD. Tutarel et al. (21) reported that of 375 ACHD aged $64.8 \pm 5.9$ years, 55 died during a median follow-up of 5.5 years. The strongest prognostic factors for mortality were CAD, symptoms of heart failure, New York Heart Association (NYHA) class, and moderate to severe reduction in systemic ventricular systolic function. Johnson et al. (22) reported that 16 out of 73 (22\%) ACHD who were referred for CAD screening prior to CHD surgery did in fact have CAD. ACHD with CAD were more likely to be older and have a history of hypertension, dyslipidemia, and tobacco smoking.

All in all, the prevalence of significant CAD among ACHD was similar to that in the general population. Traditional cardiovascular risk factors for CAD also applied to ACHD, in whom primary prevention of CAD was similarly important.

\section{Low prevalence of CAD in adults with cyanotic CHD}

In 2004, a series by Perloff (2) revealed minimal or absent signs of atherosclerosis on coronary angiography in 25 cyanotic women and 24 cyanotic men with mean ages of $43 \pm 4$ years and $41 \pm 6$ years, respectively. A more recent series in 2009 describing 250 patients in the UK who underwent selective coronary angiography for reasons other than suspected CAD revealed that the prevalence of significant CAD in a hospital ACHD cohort (9.2\%) was similar to that in the general population. No patient younger than 40 years old had significant CAD. Systemic arterial hypertension and hyperlipidemia were strong 
predictors of CAD. However, none of the cyanotic patients included had significant CAD (3). Moon et al. (23) reported that adults with cyanotic CHD had a significantly lower risk of high lipid levels, including low density lipoprotein (LDL) cholesterol, hyperlipidemia, diabetes, hypertension, and metabolic disorder, than surgically corrected ACHD and healthy controls. This result is consistent with the findings of Martínez-Quintana et al. that patients with cyanosis had significantly lower levels of total cholesterol and LDL cholesterol than those without cyanosis (24). Hypocholesterolemia can be explained by the genetic determinants of cyanosis, hypoxemia, erythrocytosis, and related factors. Regardless of the theoretical risk of atherosclerosis in ACHD, most adults with cyanotic CHD had a minimal incidence of CAD (1-3,7-10).

\section{Coronary circulation in adults with cyanotic CHD}

The coronary arteries were tortuous and markedly dilated in $15 \%$ of the adults with inherently cyanotic CHD, according to coronary arteriography $(2,11,12)$. Mild to moderate dilatation of the extramural coronary arteries in cyanotic CHD occurs in response to a collaboration between endothelial vasodilator nitric oxide and prostaglandins. This collaboration is provoked by increased endothelial shear stress of the erythrocytotic perfusate $(2,25,26)$, which also affects the proliferation of systemic vascular bed. Marked dilatation is due to medial structural degeneration that weakens the coronary artery walls and acts in concert with vasodilatation induced by endothelial nitric oxide synthase $(10,25,27)$. At the same time, angiogenesis in the lung field can develop from the coronary artery, ascending aorta, subclavian artery to pulmonary arteries, and other collateral arteries, such as pulmonary artery to pulmonary vein collaterals, venous-venous collaterals, and other multiple collaterals, including peri-coronary arterial collaterals, due to vascular endothelial growth factor and other growth factors that are seen at elevated levels in adults with cyanotic CHD (28).

\section{Factors related to the lower incidence of coronary atherosclerosis in adults with cyanotic CHD}

Numerous physiological characteristics seen in adults with cyanotic CHD may be responsible for the low incidence of atherosclerosis in this population. Hypoxemia, hypocholesterolemia, upregulation of nitric oxide levels, hyperbilirubinemia, and low platelet counts are protective factors against atherosclerosis in adults with cyanotic CHD $(9,10)$.

Hypoxemic erythrocytotic residents of high altitudes show low coronary atherosclerosis and serum cholesterol levels $(2,29)$. Likewise, cyanotic CHD is one of the causes of hypocholesterolemia, and reduced levels of both total cholesterol and LDL cholesterol (11). Decreased total cholesterol is related to low intima-medial thickness, as has been demonstrated in the general population (30). The etiology of low intima-medial thickness includes cyanosis (systemic arterial hypoxemia), erythrocytosis, and genetic factors (11). Persistence of hypocholesterolemia after surgical elimination of cyanosis, hypoxemia, and erythrocytosis implies the induction or suppression of hypocholesterolemia-inducing genes. Low to normal levels of high-density lipoprotein (HDL) cholesterol have also been noted in adults with cyanotic CHD (11). Furthermore, a number of rare genetic disorders may be associated with low HDL cholesterol levels. On the other hand, the low HDL cholesterol levels may be related to the limited exercise often seen in adults with cyanotic CHD.

In contrast to the above-sited studies, a recent report on the prevalence of subclinical atherosclerosis in a larger population of adults with cyanotic CHD found no significant differences between cyanotic CHD patients and controls in coronary artery calcification score, carotid plaques, carotid plaque thickness max, or carotid intima media thickness (31). There were also no significant differences in lipoprotein concentrations.

Hypoxemia is associated with a decrease in atherogenic oxidized plasma LDL cholesterol and a decrease in intimal oxidized LDL cholesterol. Larger LDL cholesterol particles are relatively resistant to oxidation, and the lack of small, dense, oxidation-sensitive LDL cholesterol in adults with cyanotic CHD may function in a similar manner $(2,11)$. A relationship between oxygen saturation and atherosclerosis in vascular beds is relevant. In the normal pulmonary circulation, atherosclerosis is common, especially after 40 years of age, despite hypoxemia (2). Even when oxygen saturation in the pulmonary bed is increased by a left-toright shunt, the prevalence of atherosclerosis remains the same or increases slightly compared with those without a left-to-right shunt (2).

Nitric oxide is an antiatherogenic factor because, as a paracrine molecule, it opposes platelet adherence and aggregation, stimulates disaggregation of preformed platelet aggregates, inhibits monocyte adherence and infiltration, inhibits smooth muscle cell proliferation, and 
turns off transcription of intercellular adhesion molecule-1 (ICAM-1), which governs adhesion of monocytes to the endothelium (25). The bioavailability of nitric oxide, a signaling molecule synthesized from $\mathrm{L}$ arginine and oxygen, is increased in adults with cyanotic CHD. This is due to increased endothelial shear stress induced by the viscous erythrocytotic perfusate, which is a major factor in inducing gene expression of nitric oxide and endothelial nitric oxide synthase $(2,25,32)$. Nitric oxide exerts its anti-atherogenic effects through countering platelet aggregation, stimulating disaggregation of preformed platelet aggregates, and inhibiting monocyte adherence and infiltration $(10,32)$. In addition, red blood cells are nitric oxide reservoirs and red cell mass is increased in adults with cyanotic CHD (33).

Hyperbilirubinemia is frequently observed in adults with cyanotic CHD due to secondary erythrocytosis. This is because bilirubin is formed from the breakdown of heme, a process made excessive by the increased red cell mass seen in adults with cyanotic CHD $(2,34)$. Bilirubins are endogenous antioxidants that inhibit LDL cholesterol oxidation and reduce atherosclerotic risk $(32,35)$. Gilbert's disease, a benign hereditary disorder of hepatic bilirubin metabolism, is accompanied by high levels of unconjugated bilirubin and immunity from coronary atherosclerosis (35).

Low platelet counts are antiatherogenic $(10,36)$, and platelet counts are typically low or thrombocytopenic in adults with cyanotic CHD. This is because whole megakaryocytes are shunted from the systemic venous circulation into the systemic arterial circulation, so that they cannot shed platelets by cytoplasmic fragmentation in the pulmonary vascular bed. Platelet counts correlate negatively with the magnitude of the right-to-left shunt. Although not risk factors for atherosclerosis, thrombocytopenia and secondary erythrocytosis are frequently observed in severely cyanotic patients. Contrary to the increased bleeding tendency caused by thrombocytopenia, hyperviscosity increases thrombotic risk (34). We noted this increased hyperviscosity by demonstrating significantly increased hemoglobin, hematocrit, and erythrocyte levels in adults with cyanotic CHD (2).

\section{Risk factors for CVD in different population of ACHD}

CVD in ACHD is as prevalent as in the general population, and has become a leading cause of mortality. Furthermore, specific populations of ACHD have high-risk lesions that are associated with an increased risk of CVD complications.
These ACHD may therefore warrant intensified screening and treatment (37). The risk of premature atherosclerotic CVD in ACHD is mainly based on two types of background mechanisms: lesions with coronary artery abnormalities, such as origin of the left main coronary artery from the right sinus of Valsalva, and obstructive lesions of the left ventricle and aorta, such as COA or supra-aortic stenosis. In addition, certain surgical repair procedure for CHD, like the Ross procedure or Jatene procedure, may result in abnormalities of the coronary arteries. Cardiovascular risk may also vary with the type of CHD. Specific conditions in which the coronary arteries are directly affected or surgically altered may confer greater risk for premature atherosclerotic CAD. Coronary artery re-implantation at the time of arterial switch repair in dextro-transposition of the great arteries (dTGA) was reported to result in abnormal coronary flow reserve, and intracoronary ultrasound revealed that some patients develop intimal proliferation, a precursor to atherosclerosis (38). Left-sided obstructive lesions may also be associated with accentuated cardiovascular risk. COA, even after repair, is commonly associated with systemic hypertension, and aortic stenosis can be associated with left ventricular hypertrophy and diastolic dysfunction, known risk factors for adult-onset cardiovascular morbidity and mortality (7). Furthermore, aortopathy (aortic root dilatation and stiffness of the aorta) with pathophysiological aortic medial degeneration can have a negative influence on coronary artery flow in ACHD, and that may induce CAD with advanced age (39). Aortopathy is observed in patients with Marfan syndrome, bicuspid aortic valve, TOF, dTGA, hypoplastic left heart syndrome, and other CHD $(40,41)$.

\section{Coronary artery anomalies and post-coronary artery re- implantation surgery: anomalous origin of a coronary artery from a different sinus}

The origin of the left main coronary artery from the right sinus of Valsalva is associated with sudden death, particularly during or immediately after strenuous exercise (42). In these patients, autopsies may reveal subendocardial scars and, occasionally, large myocardial infarction. Atherosclerosis in a segment of the abnormal artery has been demonstrated even in young individuals with this disorder (43-46). Six out of eight patients with both the right coronary artery and left coronary artery originating from one aortic sinus exhibited significant narrowing of the coronary artery due to atherosclerotic plaque (44). In addition, symptomatic patients with a single coronary artery 
and ischemia nearly always have associated atherosclerosis. Click et al. (43) reported that stenosis is significantly greater in an anomalous circumflex artery than in a non-anomalous circumflex artery in control subjects matched for age, gender, symptoms, and degree of atherosclerosis of nonanomalous coronary arteries. The unusual angle of takeoff and more tortuous course of the proximal portion of the anomalous coronary artery predisposes it to accelerated atherosclerosis. This is because the junction point of the bound portion of the anomalous artery and the free portion as it wraps around the aorta is an area susceptible to lipid accumulation.

Previous studies, as described above, suggested a predilection for accelerated atherosclerosis in some forms of coronary artery anomalies, whereas other studies $(47,48)$ found no increased incidence of atherosclerosis associated with such anomalies. All in all, the prevailing opinion is that coronary segments with an anomalous course are no more vulnerable to obstructive disease than normal segments in the same individual (47). Diez et al. (48) used coronary angiography to examine coronary anomalies, and found that they were more common in women, but overall, 57 of 110 patients with coronary anomalies (52\%) had no obstructive disease. Conversely, patients without obstructive disease had an increased incidence of coronary anomalies ( $8.6 \%$ versus $4.9 \%)$.

\section{Post-coronary artery re-implantation surgery; $d T G A$ after arterial switch operation, Ross procedure, and anomalous origin of left coronary artery from the pulmonary artery (Bland-White-Garland syndrome)}

Coronary manipulation is necessary in the arterial switch operation for dTGA and anomalous origin of the left coronary artery from the pulmonary artery. Coronary ostial stenosis or coronary artery kinking may progress over time, and there may be an increased risk of associated atherosclerosis (43). In patients with dTGA after arterial switching, intravascular ultrasound revealed coronary intimal thickening/early coronary atherosclerosis in translocated coronary arteries (49). Also, five years after undergoing an arterial switch operation for dTGA, the patients showed significantly less epicardial coronary dilatation in response to nitroglycerin. Further, coronary arterial flow reserve was reduced after acetylcholine administration (50). Nearly one-third of patients who underwent arterial switching or the Ross procedure were obese or overweight. Pasquali et al. (38) reported that obese arterial switch operation patients, in comparison with normal-weight atrial switch operation patients and controls, had a higher systolic blood pressure, night-time diastolic blood pressure load, and left ventricular mass index, as well as lower brachial artery reactivity. Also, in obese arterial switch operation patients, the carotid intima-media thickness was slightly increased, and significantly higher triglyceride and lower HDL cholesterol levels were seen. Moreover, Pasquali et al. (51) reported that nearly one-third of patients undergoing an arterial switch operation or Ross procedure were obese or overweight. Obese/overweight status was not related to activity restriction or early feeding practices, but was associated with parental obesity. Obese or overweight patients had a higher left ventricular mass. Obesity and associated comorbidities may pose additional cardiovascular risk in this unique population who underwent coronary artery reimplantation in childhood.

\section{Obstructive lesions of the left ventricle and aorta: coarctation of the aorta}

One of the CHDs associated with an increased risk of CVD in adulthood is obstructive lesions of the left side of the heart.

Cohen et al. (52) reported that, of 571 COA patients in long-term follow-up, $11 \%$ required subsequent cardiovascular surgery, 25\% developed hypertension, and 87 succumbed late death. The most common cause of late death was CAD in 32 patients, followed by sudden death, heart failure, cerebrovascular accidents, and ruptured aortic aneurysm. COA, even after complete repair, is commonly associated with systemic hypertension and exercise-induced hypertension, which is related to the pathophysiology for acquired CVD such as early onset atherosclerosis $(53,54)$. Arterial abnormalities may persist after COA repair and result in long-term systemic hypertension and increased risk for CVD. Hypertension is related to constriction of the aorta at the site of repair, but COA may be associated with abnormal vascular reactivity, arterial wall abnormality (aortic medial degeneration/ aortopathy), or baroreceptor dysfunction (55-57). The prevalence of hypertension at rest after repair of COA is at least 10\% (58). Exerciseinduced systolic hypertension may also develop in patients after repair of COA, even when their blood pressure is normal at rest (59). Beyond hypertension, COA is associated with other important sequelae that lead to morbidity and mortality. Cerebrovascular accidents occur in association with systemic hypertension, especially in 
patients with cerebral arterial aneurysms, $10 \%$ of whom have accompanying COA (60). Aortic dissection in the ascending aorta or near the COA repair site may also develop (41). Persistent hypertension, association with bicuspid aortic valve, aortic atherosclerosis, and dilated aorta (aortopathy) predispose COA patients to serious cardiovascular risk $(53,54)$. Persistent hypertension, older age at repair, association with bicuspid aortic valve, aortic atherosclerosis, and dilation of the aorta proximal to the repair site all predispose coarctation patients to this serious risk (59). Roifman et al. (61) compared 756 patients with COA and 6,481 with ventricular septal defects, and found that $37 / 756(4.9 \%)$ and $224 / 6,481(3.5 \%)$ had a history of CAD, respectively. Male sex, hypertension, diabetes mellitus, and hyperlipidemia all independently predicted the development of CAD. COA did not independently predict the development of CAD or premature CAD. Although traditional cardiovascular risk factors did independently predict the development of CAD, the diagnosis of COA alone did not. Fedchenko et al. (62) conducted comprehensive cardiovascular risk assessment of 72 adults with COA (age 43.5 years). Sixty-six (91.7\%) patients had at least one cardiovascular risk factor and $40.3 \%$ had three or more risk factors. More than half of the patients had hyperlipidemia $(\mathrm{n}=42,58.3 \%)$ and 35 patients $(48.6 \%)$ were overweight or obese. Of the 60 patients who underwent 24-hour ambulatory blood pressure measurement, 33 (55.0\%) were hypertensive. Cardiovascular risk factors among patients with COA are prevalent. This suggests a need for more aggressive screening strategies for traditional risk factors to minimize the risk of these patients also developing atherosclerotic disease.

\section{Obstructive lesions of the left ventricle and aorta: aortic stenosis}

Another obstructive lesion of the left side of the heart in aortic stenosis. Aortic stenosis develops most often at the level of the aortic valve, but can develop at supravalvular and subvalvular regions, resulting in myocardial damage and CVD. Valvular aortic stenosis occurs in 3\% to $6 \%$ of patients with CHD (63). Aortic stenosis associated with left ventricular hypertrophy and diastolic dysfunction is a known risk factor for adult-onset CVD mortality and morbidity (64). Moreover, myocardial blood flow may be compromised in patients with aortic stenosis despite normal coronary artery patency. Increased myocardial work results in increased demand for oxygen, exceeding the capacity of the coronary supply (abnormal coronary flow reserve). Aortic stenosis during childhood can progress and may therefore be associated with increased left ventricular mass and increased risk for CVD as adults. Increases in the left ventricular outflow tract gradient are heightened by progressive calcification of the aortic valve (63). Supravalvular aortic stenosis [most commonly associated with Williams syndrome (65)] is associated with coronary ostial stenosis and hypertension in the ascending aorta and subsequent coronary arterial stenosis, and may result in myocardial ischemia and exercise-induced syncope. Aortic stenosis-associated renal arterial stenosis can also induce systemic hypertension $(53,66)$.

\section{Aortopathy and CAD}

The aorta in some types of CHD, such as bicuspid aortic valve, COA, and TOF, among others, can dilate, become aneurysmal, and may rupture $(27,41)$. In adults with these types of CHD, reduced aortic elasticity and increased stiffness of the dilated aorta can induce aortic regurgitation, reduce coronary artery flow, and negatively impact left ventricular systolic and diastolic function (left ventricular hypertrophy and failure). In this way, aortopathy in ACHD can have an unfavorable influence on coronary artery flow and may induce CAD with advanced age (41). Senzaki et al. (67) describe abnormal ventriculoarterial coupling (increased aortic stiffness) in repaired TOF. They found that TOF patients $(38$ repaired TOF patients vs. 55 controls) had a higher impedance, pulse wave velocity, and arterial wave reflection, and lower total peripheral arterial compliance, in addition to increased aortic wall stiffness and increased aortic root diameter, compared with the controls. Central and peripheral arterial wall stiffness are increased even after TOF repair. Abnormal arterial elastic properties in repaired TOF induce increased left ventricular afterload, decreased coronary artery flow, and aortic dilatation, and these may develop into systolic and diastolic dysfunction of the left ventricle.

\section{Causes of death in ACHD}

The mortality of ACHD is continuing to decline, and their causes of death are changing. Among adults with cyanotic lesions, such as TOF and dTGA after repair, the primary cause of death was arrhythmia, followed by heart failure (68). However, for adults with noncyanotic lesions, the major cause before 1990 was arrhythmia; after 1990, myocardial 
infarction became the leading cause of death in ACHD in the US. Myocardial infarction is now the leading cause of death for adults with noncyanotic CHD worldwide, consistent with the long survival and increasing impact of acquired heart disease. However, arrhythmia remains the primary cause of death for those with cyanotic lesions. Whether the leading cause of death will change for adults with cyanotic CHD in the near future remains unknown because of its antiatherogenic properties.

\section{Metabolic syndrome and risk factors for CAD in ACHD}

ACHD are at risk of developing atherosclerotic CVD. Empirical evidence further suggests that ACHD are prone to developing CAD. The prevalence of cardiovascular risk factors in these patients is similar to that in the general population $(6,69,70)$.

\section{Metabolic syndrome}

Metabolic syndrome is a constellation of risk factors for CVD, including obesity, dyslipidemia, insulin resistance, and hypertension. These risk factors are associated with excessive acquired CVD and type 2 diabetes mellitus, as well as increased mortality in general $(14,25)$. According to one study on CVD risks in ACHD (71), the prevalence of metabolic syndrome in ACHD in Japan was 16\%; far greater than the $5.5 \%$ prevalence of metabolic syndrome in the general population in Japan (NCEP ATP III) (72). In the US, Deen et al. (13) also report that metabolic syndrome was more common in ACHD than in controls (15.0\% vs. $7.4 \%$ in 448 ACHD and 448 controls, respectively). However, in Germany, Häcker et al. (73) found that 72 out of 512 ACHD aged $43.0 \pm 9.6$ years $(14.1 \%)$ had metabolic syndrome, compared with $21.5 \%$ in a 40 - to 49 -yearold cohort from a general population-based sample (74). Regarding severity class, patients with simple, moderate, and severe forms of ACHD had a metabolic syndrome prevalence of $11.8 \%, 16.7 \%$, and $13.8 \%$, respectively. In Korea, Moon et al. (23) reported that adults with repaired acyanotic CHD had a higher rate of hypertension, diabetes mellitus, and hypercholesterolemia than those with cyanotic CHD and healthy controls. Metabolic syndrome had the highest distribution in the adults with surgically corrected CHD (1.48 times higher than in the controls). Looking more closely at the individual components of metabolic syndrome, subjects in the adults with surgically corrected
CHD group had higher blood pressure, higher fasting blood sugar, higher triglycerides, lower HDL cholesterol, and higher rates of obesity than adults in the cyanotic CHD group and control groups. This suggests that metabolic syndrome is more common among adults with acyanotic CHD than in the general population.

ACHD should be screened for metabolic syndrome and risk factors to prevent atherosclerotic CAD. A sedentary lifestyle may be an independent risk factor for accelerated atherosclerosis and obesity. Therefore, preventive care for metabolic syndrome should include healthy lifestyle counseling, blood pressure monitoring, and screening for lipid abnormalities and insulin resistance. Cardiac rehabilitation has been demonstrated to improve the exercise performance of children with CHD, even those with known residual cardiac dysfunction (75). After proper exercise capacity evaluation, most ACHD should be able to perform appropriate aerobic activities. In addition, pediatric cardiologists have an important role to play in preventive cardiology counseling of children with CHD during the transition process, because atherosclerosis is known to start during childhood.

\section{Overweight/obesity}

In a study of 224 Japanese ACHD, it was reported that the prevalence of obesity [body mass index (BMI) $\geq 30 \mathrm{~kg} / \mathrm{m}^{2}$ ] was higher in ACHD than in the general Japanese population $(10.1 \%$ vs. $2.9 \%)(71,76)$. In a previous report, the prevalence of obesity and overweight in ACHD was similar to that in the general national population (77). In one published study, $26 \%$ of 1,532 children with CHD were overweight or obese (7). In a study from Belgium, the prevalence of obesity and hypertension in ACHD was higher than that in the general population (6). Furthermore, obesity was present in $30 \%$ of adults with moderate and complex CHD requiring surgery (5). Nearly one-third of patients with a history of dTGA after an arterial switch operation were obese (38). In previous studies on patients with single ventricle who had undergone Fontan procedure, obesity was present in $11 \%$ of pediatric patients and $17 \%$ of adult patients $(78,79)$. Several recent studies report that patients who have undergone Fontan procedure have an increased prevalence of obesity (80-82). In contrast, Zaqout et al. (83) collected data on 539 ACHD and found that men with a ventricular septal defect, COA, and Fontan circulation, and women with Fontan circulation had a lower average body weight than their reference group. Being 
underweight was more prevalent in women with severe lesions than in the reference group (22.2\% vs. 3.8\%).

The association of obesity with CHD may complicate the management of ACHD, who are already at risk for ventricular dysfunction, arrhythmias, and heart failure $(84,85)$. Obesity is independently associated with hypertension and endothelial dysfunction. One of the contributing factors for obesity in ACHD, especially in those with moderate to severe ACHD, is physical inactivity. ACHD are frequently self-restrictive for exercise $(86,87)$. Moreover, as many grew up with a desire to gain weight early in life when weight gain was poor, they may be affected by high-caloric supplemental diets. ACHD possess unique risk factors for developing obesity, including exercise restriction and differing nutritional strategies in infancy and early childhood $(6,77,88,89)$. Although restrictions on competitive sports are recommended for certain high-risk populations, most patients with repaired CHD may exercise safely; however, medical providers and parents often impose unnecessary exercise restrictions (90). Increased physical activity and aerobic exercise play an important role in reducing cardiovascular risk. However, questions still remain regarding this effect in ACHD. Similar to the general population, obesity is a major risk factor for the development of metabolic syndrome in ACHD (91). Similar growth patterns seen in infants without CHD are associated with obesity and a greater risk of adult CVD $(92,93)$. Lerman et al. recently reported that, compared with controls, ACHD had a similar prevalence of overweight and obesity, but a lower prevalence of morbid obesity (94). Although they had a higher incidence of obesity, some ACHD, especially those with complex CHD or cyanotic CHD, had sarcopenia (95), and they had higher intakes of calories, protein, and fat than those in a national survey, despite decreased skeletal muscle mass. Amino acid intake plus resistance training may improve the body fat percentage and skeletal muscle mass in ACHD.

\section{Hypertension}

Systolic blood pressure has been found to be higher in overweight and obese ACHD than in those of normal weight $(7,53)$. Mechanistically increased afterload due to hypertension can induce left ventricular hypertrophy, which leads to increased left ventricular mass, and these factors adversely affect the delicate physiology of complex CHD (e.g., Fontan or systemic right ventricular physiology) as well as underlying CHD with valvular regurgitation and ventricular dysfunction. Furthermore, systemic hypertension is another important cardiovascular risk factor that can lead to premature CAD, stroke, arrhythmia, and CVD. The prevalence of hypertension in ACHD (71) is lower than that in the general Japanese population (15.1\% vs. $26.5 \%$ in males). A recent study on blood pressure in 100 ACHD (age, 37.0 \pm 15.0 years) (96) reported an average blood pressure of $117.7 \pm 20.1 / 69.7 \pm 14.3 \mathrm{mmHg}$, with a central blood pressure of $119.9 \pm 21.5 \mathrm{mmHg}$. Hypertension was observed in 16 (16\%). The determinants of high systolic blood pressure were age, hemoglobin A1c, and total cholesterol. High systolic blood pressure is common in ACHD; therefore, monitoring and strict blood pressure control are essential. In the Belgian study described above, $13 \%$ of ACHD were hypertensive (6). In an older population of ACHD (age $>65$ years), $47 \%$ were hypertensive (97); however, many of them had simple CHD. In general, hypertension in this population is essential hypertension. There are, however, patients with moderate or complex CHD who have substantially increased risk of hypertension, including COA. Although hypertension was noted in only $4 \%$ of the CHD group as a whole, as many as $46 \%$ of patients became hypertensive after COA repair (98). Hypertension may increase the risk of aortic dilation in ACHD prior to COA repair, bicuspid aortic valve, TOF, or dTGA after arterial switch operation. However, the effects of longstanding hypertension on ACHD remain unknown.

\section{Dyslipidemia}

In one study of ACHD (71), lipid profiles were within normal limits: mean total cholesterol was $177 \mathrm{mg} / \mathrm{dL}$, mean LDL cholesterol was $101 \mathrm{mg} / \mathrm{dL}$, mean HDL cholesterol was $54 \mathrm{mg} / \mathrm{dL}$, and mean triglycerides level was $126 \mathrm{mg} / \mathrm{dL}$. There were no differences between the cyanotic and acyanotic CHD groups. In another report on CHD patients and serum cholesterol levels (99), children with cyanotic CHD had lower cholesterol levels than those with acyanotic CHD and arrhythmia patients, and this tendency increased with age. There is no evidence to suggest that the prevalence of dyslipidemia is higher in ACHD than in the general population $(4,100)$. One UK single-center study of 250 ACHD (mean age 51 years) reported the prevalence of dyslipidemia to be $19.1 \%$, with dyslipidemia being a strong risk factor for CAD (3). The prevalence of dyslipidemia, however, decreased with increasing cardiac lesion complexity. Data from the Quebec Congenital Heart Disease Database revealed the prevalence of dyslipidemia 
to be $27 \%$ in patients over the age of 65 , which is lower than estimates for the general population (101). Adults with cyanotic CHD have lower total cholesterol than acyanotic patients $(10,11)$. This effect persists even in patients surgically corrected to have acyanotic circulation (11). Martinez-Quintana et al. reported that ACHD had lower total cholesterol and LDL cholesterol levels than nonCHD patients, although no significant differences were noted in lipoprotein (a) concentrations between the groups $(24,102)$. Adults with cyanotic CHD have significantly lower total and LDL cholesterol levels than non-CHD patients; therefore, they may have a lower risk of developing atherosclerosis in the future $(8,10)$.

\section{Type 2 diabetes mellitus and impaired fasting glucose levels}

Ohuchi et al. reported lower fasting glucose, but higher postprandial blood glucose and glycated hemoglobin levels, in adult patients with cyanotic CHD and Fontan patients, compared with healthy controls (103). Dellborg et al. (104) reported that CHD frequently coexists with secondary risk factors for CVD, and the development of type 2 diabetes mellitus in the ACHD population is not uncommon, with an estimated prevalence of 4 to $8 \%$ in Sweden. In ACHD, the prevalence of type 2 diabetes mellitus and impaired fasting glucose were $9.2 \%$ and $5.9 \%$, respectively, and there was no significant difference between those with cyanotic and acyanotic CHD (71). Martinez-Quintana et al. (24) identified an increased risk of metabolic dysfunction in ACHD, consistent with decreased insulin sensitivity. These study results are consistent with previously published findings of high abnormal glucose tolerance and low HDL cholesterol levels in ACHD $(24,103)$. This pathophysiology should be taken into account for long-term follow-up of adults with complex CHD (103). These ACHD also exhibited activated neurohormonal activities, abnormal cardiac autonomic nervous activity, and inflammatory cytokines, similar to those demonstrated in non-CHD adults with heart failure (105). The treatment of conventional cardiovascular risk factors in ACHD can be considered secondary prevention, considering the relatively high morbidity and risk of mortality in adult patients with the combination of CHD and type 2 diabetes mellitus. However, a prognostic value for abnormal glucose regulation remains unknown, especially regarding prognosis for mortality in ACHD.

\section{Recommendations for ACHD to prevent the development of metabolic syndrome, atherosclerosis, and CVD}

Previous studies have demonstrated that the prevalence of cardiovascular risk factors, such as obesity and metabolic syndrome, is higher in ACHD than in the general population. Repaired ACHD may lead to premature atherosclerosis. Many children with repaired CHD have limited physical activity during school. A life style of avoiding exercise or walking is an independent risk factor for atherosclerosis. Moreover, ACHD are more prone to obesity. As one means to address this, cardiac rehabilitation has been reported to improve the exercise performance of ACHD, even those with known residual cardiac dysfunction. As ACHD have other abnormalities that may make the heart more vulnerable to both the development of atherosclerosis and the adverse sequelae of a cardiovascular event, evaluation of their CVD risk status should be detailed. This is particularly true for ACHD who have risk factors for developing CVD. Adults with certain specific types of CHD patients are at risk for premature CVD. Even in adults with cyanotic CHD, regardless of repair, eliminating risk factors for CVD is essential. Preventive and therapeutic measures for primary and secondary prevention of cardiovascular events should be applied to this special group of patients.

\section{Conclusions}

Metabolic syndrome has a higher prevalence among ACHD than in the general population. The prevalence of CAD is similar in adults with acyanotic CHD and in the general population. Adults with cyanotic CHD, even after repair, have a lower incidence of CAD than those with acyanotic CHD. However, even those with cyanotic CHD can develop CAD when they have metabolic syndrome or risk factors such as obesity, dyslipidemia, hypertension, diabetes mellitus, smoking habit, and/or a positive family history of CVD, together with limited exercise. The prevalence of risk factors for CVD is similar between ACHD and the general population. An increased risk of coronary atherosclerosis is observed in congenital coronary artery anomalies, dTGA after arterial switch operation, Ross procedure, and COA. Aortopathy may be an additional risk factor for CVD. As ACHD have other abnormalities that may make the heart 
more vulnerable to both the development of atherosclerosis and adverse sequelae of a cardiovascular event, it may be reasonable to regularly evaluate their CVD risk status. This is especially true for ACHD with specific types of CHD that confer higher risk; therefore, all ACHD should be monitored closely, regardless of having risk factors for CAD.

\section{Acknowledgments}

Funding: None.

\section{Footnote}

Provenance and Peer Review: This article was commissioned by the editorial office, Cardiovascular Diagnosis and Therapy for the series "Current Management Aspects in Adult Congenital Heart Disease (ACHD): Part III". The article has undergone external peer review.

Conflicts of Interest: The author has completed the ICMJE uniform disclosure form (available at http:// dx.doi.org/10.21037/cdt-20-781). The series "Current Management Aspects in Adult Congenital Heart Disease (ACHD): Part III" was commissioned by the editorial office without any funding or sponsorship. $\mathrm{KN}$ serves as an unpaid editorial board member of Cardiovascular Diagnosis and Therapy from Oct 2018 to Sep 2020 and served as the unpaid Guest Editor of the series. The author has no other conflicts of interest to declare.

Ethical Statement: The author is accountable for all aspects of the work in ensuring that questions related to the accuracy or integrity of any part of the work are appropriately investigated and resolved.

Open Access Statement: This is an Open Access article distributed in accordance with the Creative Commons Attribution-NonCommercial-NoDerivs 4.0 International License (CC BY-NC-ND 4.0), which permits the noncommercial replication and distribution of the article with the strict proviso that no changes or edits are made and the original work is properly cited (including links to both the formal publication through the relevant DOI and the license). See: https://creativecommons.org/licenses/by-nc$\mathrm{nd} / 4.0 /$.

\section{References}

1. Niwa K. Adults with congenital heart disease transition Curr Opin Pediatr 2015;27:576-80.

2. Perloff JK. The Coronary Circulation in Cyanotic Congenital Heart Disease. Int J Cardiol 2004;97:79-86.

3. Giannakoulas G, Dimopoulos K, Engel R, et al. Burden of coronary artery disease in adults with congenital heart disease and its relation to congenital and traditional heart risk factors. Am J Cardiol 2009;103:1445-50.

4. Yalonetsky S, Horlick EM, Osten MD, et al. Clinical characteristics of coronary artery disease in adults with congenital heart defects. Int J Cardiol 2013;164:217-20.

5. Zaidi AN, Bauer JA, Michalsky MP, et al. The impact of obesity on early postoperative outcomes in adults with congenital heart disease. Congenit Heart Dis 2011;6:241-6.

6. Moons P, Deyk KV, Dedroog, D, et al. Prevalence of cardiovascular risk factors in adults with congenital heart disease. Eur J Cardiovasc Prev Rehabil 2006;13:612-6.

7. Pemberton VL, McCrindle BW, Barkin S, et al. Report of the National Heart, Lung, and Blood Institute's Working Group on Obesity and Other Cardiovascular Risk Factors in Congenital Heart Disease. Circulation 2010;121;1153-9.

8. Duffels MG, Mulder KM, Trip MD, et al. Atherosclerosis in patients with cyanotic congenital heart disease. Circ J 2010;74:1436-41.

9. Niwa K. The coronary circulation on adults with congenital heart disease. Intern Med 2006;45:1199-200.

10. Perloff JK. Cyanotic congenital heart disease the coronary arterial circulation. Curr Cardiol Rev 2012;8:1-5.

11. Fyfe A, Perloff JK, Niwa K, et al. Cyanotic congenital heart disease and coronary atherogenesis. Am J Cardiol 2005;96:283-90.

12. Chugh R, Perloff JK, Fishbein M, et al. Extramural coronary arteries in adults with cyanotic congenital heart disease. Am J Cardiol 2004;94:1355-7.

13. Deen JF, Krieger EV, Slee AE, et al. Metabolic syndrome in adults with congenital heart disease. J Am Heart Assoc 2016;5.:e001132.

14. Mottillo S, Filion KB, Genest J, et al. The metabolic syndrome and cardiovascular risk a systematic review and meta-analysis. J Am Coll Cardiol 2010;56:1113-32.

15. Giamberti A, Lo Rito M, Conforti E, et al. Acquired coronary artery disease in adult patients with congenital 
heart disease: a true or a false problem? J Cardiovasc Med (Hagerstown) 2017;18:605-9.

16. Stulak JM, Dearani JA, Burkhart HM, et al. Coronary artery disease in adult congenital heart disease: outcome after coronary artery bypass grafting. Ann Thorac Surg 2012;93:116-22.

17. Bokma JP, Winter MM, Kuijpers JM, et al. Role of acquired cardio- vascular disease in tetralogy of Fallot patients $>50$ years of age. J Am Coll Cardiol 2017;69:2465-6.

18. Egbe AC, Ananthaneni S, Jadav R, et al. Coronary artery disease in adults with tetralogy of Fallot. Congenital Heart Disease 2019;14:491-7.

19. Bokma JP, Zegstroo I, Kuijpers JM, et al. Factors associated with coronary artery disease and stroke in adults with congenital heart disease. Heart 2018;104:574-80.

20. Bauer UMM, Körten MA, Diller GP, et al. Cardiovascular risk factors in adults with congenital heart defects Recognised but not treated? An analysis of the German National Register for Congenital Heart Defects. Int J Cardiol 2019;277:79-84.

21. Tutarel O, Kempny A, Alonso-Gonzalez R, et al. Congenital heart disease beyond the age of 60 : emergence of a new population with high resource utilization, high morbidity, and high mortality. Eur Heart J 2014;3 5:725-32.

22. Johnson B, Buelow M, Earing M, et al. Coronary artery disease screening in adults with congenital heart disease prior to surgery. Congenit Heart Dis 2019;14:895-900.

23. Moon JR, Song J, Huh J, et al. Analysis of cardiovascular risk factors in adults with congenital heart disease. Korean Circ J 2015;45:416-23.

24. Martínez-Quintana E, Rodríguez-González F, Nieto-Lago $\mathrm{V}$, et al. Serum glucose and lipid levels in adult congenital heart disease patients. Metabolism. 2010;59:1642-8.

25. Malinski T. Normal and pathological distribution of nitric oxide in the cardiovascular system. Pol J Pharmacol 1998; 50:387-91.

26. Koller A, Sun D, Kaley G. Role of shear stress and endothelial prostaglandins in flow and velocity-induced dilatation in vivo. Circ Res 1993:72:1276-84.

27. Niwa K, Perloff JK, Bhuta S, et al. Structural abnormalities of great arterial walls in congenital heart disease. Light and electron microscopic analysis. Circulation 2001;103:393-400.

28. Hamada H, Ebata R, Higashi K, et al, Serum vascular endothelial growth factor in cyanotic congenital heart disease functionally contributes to endothelial cell kinetics in vitro. Int J Cardiol 2007;120:66-71.

29. Arias-Stella J, Topilsky M. Anatomy of the coronary artery circulation at high altitude in high altitude physiology. Edinburgh, Chur- chill Livingstone Publishers, 1971:149-57.

30. Reiner, Z. Catapano AL, De Backer G, et al. ESC/EAS Guidelines in the Management of Dyslipidaemias. Eur Heart J 2011; 32:1769-818.

31. Tarp JB, Sørgaard MH, Christoffersen C, et al. Subclinical atherosclerosis in patients with cyanotic congenital heart disease. Int J Cardiol 2019;277:97-103.

32. Niwa K. Coronary artery anomalies and coronary artery disease in adults with congenital cardiac disease. Asia Pacific Cardiol 2007;1:70-2.

33. Gross SS, Lane P. Physiologic reaction of nitric oxide and hemoglobin. Proc Natl Acad Sci 1999;96:9967-9.

34. Niwa K, Perloff JK, Kaplan S, et al. Eisenmenger syndrome in adults: ventricular septal defect, truncus arteriosus, univentricular heart. J Am Coll Cardiol 1999;34:223-32.

35. Madhavan M, Wu LL, Hunt DC, et al. Serum bilirubin distribution and its relation to cardiovascular risks in children and young adults. Atherosclerosis 1997;131:107-13.

36. Lill MC, Perloff JK, Child JS. Pathogenesis of thrombocytopenia in cyanotic congenital heart disease. Am J Cardiol 2006;98:254-8.

37. Awerbach JD, Krasuski RA, Camitta MGW. Coronary disease and modifying cardiovascular risk in adult congenital heart disease patients: Should general guidelines apply? Prog Cardiovasc Dis 2018;61:300-7.

38. Pasquali SK, Marino BS, Powell DJ, et al, Following the arterial switch operation, obese children have risk factors for early cardiovascular disease. Congenit Heart Dis 2010;5:16-24.

39. Niwa K. Pathological background. In, Aortopathy, Niwa K, Kaemmerer H eds, Springer, Tokyo, 2017,15-30.

40. Niwa K. Landmark lecture: Perloff lecture: Tribute to Professor Joseph Kayle Perloff and lessons learned from him: aortopathy in adults with CHD. Cardiol Young 2017;27:1959-65.

41. Niwa K. Aortopathy in congenital heart disease in adults: Aortic dilatation with decreased aortic elasticity that impacts negatively on left ventricular function. Korean Circ J 2013;43:215-20.

42. Basso C, Maron BJ, Corrado D, et al. Clinical profile of congenital coronary artery anomalies with origin from the wrong aortic sinus leading to sudden death in young 
competitive athletes. J Am Coll Cardiol 2000;35:1493-501.

43. Click RL, Holmes DR Jr, Vlietstra RE, et al. Anomalous coronary arteries: location, degree of atherosclerosis and effect on survival: a report from the coronary artery surgery study. J Am Coll Cardiol 1989;13:531-7.

44. Roberts WC. Major anomalies of coronary arterial origin seen in adulthood. Am Heart J 1986;111:941-63.

45. Blake HA, Manion WC, Mattingly TW, et al. Coronary artery anomalies. Circulation 1964;30:927-40.

46. Rigatelli G, Gemelli M, Franco G, et al. Coronary artery anomalies and coronary disease: is there a relationship? Minerva Cardioangiologica 2001;49:165-8.

47. Angelini P, Velasco JA, Flamm S. Coronary anomalies: incidence, pathophysiology, and clinical relevance. Circulation 2002;105:2449-54.

48. Diez JD, Angelini P, Lee VV. Does the anomalous congenital origin of a coronary artery predispose to the development of stenotic atherosclerotic lesions in its proximal segment? Circulation 1997;96 (Suppl): I-154. Abstract.

49. Pedra SR, Pedra CA, Abizaid AA, et al. Intracoronary ultrasound assessment late after the arterial switch operation for transposition of the great arteries. J Am Coll Cardiol 2005;45:2061-8.

50. Gagliardi MG, Adorisio R, Crea F, et al. Abnormal vasomotor function of the epicardial coronary arteries in children five to eight years after arterial switch operation: an angiographic and intracoronary Doppler flow wire study. J Am Coll Cardiol 2005;46:1565-72.

51. Pasquali SK, Marino BS, Pudusseri A, et al. Risk factors and comorbidities associated with obesity in children and adolescents after the arterial switch operation and Ross procedure. Am Heart J 2009;158:473-9.

52. Cohen M, Fuster V, Steele PM, et al. Coarctation of the aorta: long-term follow-up and prediction of outcome after surgical correction. Circulation 1989;80:840-5.

53. Kavey RE, Allada V, Daniels SR, et al. Cardiovascular risk reduction in high-risk pediatric patients: a scientific statement from the American Heart Association Expert Panel on Population and Prevention Science; the Councils on Cardiovascular Disease in the Young, Epidemiology and Prevention, Nutrition, Physical Activity and Metabolism, High Blood Pressure Research, Cardiovascular Nursing, and the Kidney in Heart Disease; and the Interdisciplinary Working Group on Quality of Care and Outcomes Research: endorsed by the American Academy of Pediatrics. Circulation 2006;114:2710-38.

54. Vriend JW, van Montfrans GA, Romkes HH, et al.
Relation between exercise-insduced hypertension and sustained hypertension in adult patients after successful repair of aortic coarctation. J Hypertens 2004;22:501-9.

55. Gidding SS, Rocchini AP, Moorehead C, et al. Increased forearm vascular reactivity in patients with hypertension after repair of coarctation. Circulation 1985;71:495-9.

56. Beekman RH, Katz HP, Moorehead-Steffens C, et al. Altered baroreceptor function in children with systolic hypertension after coarctation repair. Am J Cardiol 1983;52:112-7.

57. Strafford MA, Griffiths SP, Gersony WM. Coarctation of the aorta: a study in delayed detection. Pediatrics 1982;69:159-63.

58. Brouwer RM, Erasmus ME, Ebels T, et al. Influence of age on survival, late hypertension, and recoarctation in elective aortic coarctation repair: including long-term results after elective aortic coarctation repair with a follow-up from 25 to 44 years. J Thorac Cardiovasc Surg 1994;108:525-31.

59. Freed MD, Rocchini A, Rosenthal A, et al. Exerciseinduced hypertension after surgical repair of coarctation of the aorta. Am J Cardiol 1979;43:253-8.

60. Connolly HM, Huston J 3rd, Brown RD Jr, et al. Intracranial aneurysm in patients with coarctation of the aorta: a prospective magnetic resonance angiographic study of 100 patients. Mayo Clin Proc 2003;78:1491-9.

61. Roifman I, Therrien J, Ionescu-Ittu R, et al. Coarctation of the aorta and coronary artery disease. Fact or fiction. Circulation 2012;126:16-21.

62. Fedchenko M, Mandalenakis Z, Dellborg H, et al. Cardiovascular risk factors in adults with coarctation of the aorta. Congenit Heart Dis 2019;14:549-58.

63. Roberts WC. Anatomically isolated aortic valvular disease: the case against its being of rheumatic etiology Am J Med 1970;49:151-9.

64. Levy D, Garrison RJ, Savage DD, et al. Prognostic implications of echocardiographically determined left ventricular mass in the Framingham Heart Study. N Engl J Med 1990;322:1561-6.

65. Williams JC, Barratt-Boyes BG, Lowe JB. Supravalvular aortic stenosis. Circulation 1961;24:1311-8.

66. Daniels SR, Loggie JM, Schwartz DC, et al. Systemic hypertension secondary to peripheral vascular anomalies in patients with Williams syndrome. J Pediatr 1985;106:249-51.

67. Senzaki H, Iwamoto Y, Ishido H, et al. Arterial haemodynamics in patients after repair of tetralogy of Fallot: influence on left ventricular after load and aortic dilatation. Heart 2008;94:70-4. 
68. Pillutla P, Shetty KD, Foster E. Mortality associated with adult congenital heart disease: Trends in the US population from 1979 to 2005. Am Heart J 2009;158:874-9.

69. Niwa K. Metabolic Syndrome in Adult Congenital Heart Disease. Korean Circ J 2019;49:691-708.

70. Lui, GK, Rogers IS, Ding VY, et al. Risk estimates for atherosclerotic cardiovascular disease in adults with congenital heart disease. Am J Cardiol 2017;119:112-8.

71. Javier AD, Niwa K. Cardiovascular risk factors among adults with congenital heart disease. Pediatr Cardiol Cardiac Surg (official J of Japanese Society of Pediatric Cardiology and Cardiac Surgery 2013;29 Supple:s137.

72. Expert Panel on Detection, Evaluation, and Treatment of High Blood Cholesterol in Adults. National Cholesterol Education Program: Executive summary of the third report of the National Cholesterol Education Program (NCEP) Expert Panel on Detection, Evaluation, and Treatment of High Blood Cholesterol in Adults (Adult Treatment Panel III). JAMA 2001;285:2486-97.

73. Häcker AL, Oberhoffer R, Hager A, et al. Metabolic syndrome in adults with congenital heart disease and increased intima-media thickness. Congenital Heart Disease 2019;14:945-51.

74. Neuhauser H, Ellert U. Estimation of the metabolic syndrome prevalence in the general population in Germany. J Public Health 2008;16:221-7.

75. Rhodes J, Curran TJ, Camil L, et al. Impact of cardiac rehabilitation on the exercise function of children with serious congenital heart disease. Pediatrics 2005;116:1339-45.

76. Results of National health and nutrition survey by the Japanese Ministry of Health, Labour and Welfare. Available online: https://www.mhlw.go.jp/stf/ seisakunitsuite/bunya/kenkou_iryou/kenkou/eiyou/h29houkoku.html

77. Shustak RJ, MacGuire SB, October TW, et al. Prevalence of obesity among patients with congenital and acquired heart disease. Pediatr Cardiol 2012;33:8-14.

78. Martinez SC, Byku M, Novak EL, et al. Increased body mass index is associated with congestive heart failure and mortality in adult Fontan patients. Congenit Heart Dis 2016;11:71-9.

79. Vogt KN, Manlhiot C, Van Arsdell G, et al. Somatic growth in children with single ventricle physiology impact of physiologic state. J Am Coll Cardiol 2007;50:1876-83.

80. Freud LR, Webster G, Costello JM, et al. Growth and obesity among older single ventricle patients presenting for Fontan conversion. World J Pediatr Congenit Heart Surg 2015;6:514-20.

81. Chung ST, Hong B, Patterson L, et al. High overweight and obesity in Fontan patients: a 20-year history. Pediatr Cardiol 2016;37:192-200.

82. Wellnitz K, Harris IS, Sapru A, et al. Longitudinal development of obesity in the post-Fontan population. Eur J Clin Nutr 2015;69:1105-8.

83. Zaqout M, Vandekerckhove K, Michels N, et al. Body mass index in adults with congenital heart disease. Congenital Heart Disease 2019;14:479-86.

84. Daniels SR, Kimball TR, Morrison JA, et al. Effect of lean body mass, fat mass, blood pressure, and sexual maturation on left ventricular mass in children and adolescents. Statistical, biological, and clinical significance. Circulation 1995;92:3249-54.

85. Maser RE, Lenhard MJ. An overview of the effect of weight loss on cardiovascular autonomic function. Curr Diabetes Rev 2007;3:204-11.

86. Diller GP, Dimopoulos K, Okonko D, et al. Exercise intolerance in adult congenital heart disease: comparative severity, correlates, and prognostic implication. Circulation 2005;112:828-35.

87. Reybrouck T, Mertens L. Physical performance and physical activity in grown- up congenital heart disease. Eur J Cardiovasc Prev Rehabil 2005;12:498-502.

88. Cohen MS. Clinical practice: the effect of obesity in children with congenital heart disease. Eur J Pediatr 2012;171:1145-50.

89. Stefan MA, Hopman WM, Smythe JF. Effect of activity restriction owing to heart disease on obesity. Arch Pediatr Adolesc Med 2005;159:477-81.

90. Longmuir PE, Brothers JA, de Ferranti SD, et al. American Heart Association Atherosclerosis and Obesity in Youth Committee of the Council on Cardiovascular Disease in the Y. Promotion of physical activity for children and adults with congenital heart disease: a scientific statement from the American Heart Association. Circulation 2013;127:2147-59.

91. Owens JL, Musa N. Nutrition support after neonatal cardiac surgery. Nutr Clin Pract 2009;24:242-9.

92. Barker DJ, Osmond C, Forsen TJ, et al. Trajectories of growth among children who have coronary events as adults. N Engl J Med 2005;353:1802-9.

93. Vieira TC, Trigo M, Alonso RR, et al. Assessment of food intake in infants between 0 and 24 months with congenital heart disease. Arq Bras Cardiol 2007;89:219-24.

94. Lerman JB, Parness IA, Shenoy RU. Body weights in 
adults with congenital heart disease and the obesity frequency. Am J Cardiol 2017;119:638-42.

95. Shiina Y, Matsumoto N, Okamura D, et al. Sarcopenia in adults with congenital heart disease: Nutritional status, dietary intake, and resistance training. J Cardiol 2019;74:84-9.

96. Murakami T, Shiraishi M, Shiina Y, et al. High blood pressure in adult patients with congenital heart disease. J Adult Congenit Heart D 2013;5:99.

97. Afilalo J, Therrien J, Pilote L, et al. Geriatric congenital heart disease: burden of disease and predictors of mortality. J Am Coll Cardiol 2011;58:1509-15.

98. Engelfriet P, Boersma E, Oechslin E, et al. The spectrum of adult congenital heart disease in Europe: morbidity and mortality in a 5 year follow-up period. The Euro Heart Survey on adult congenital heart disease. Eur Heart J 2005;26:2325-33.

99. Higashi K, Tateno S, Niwa K, et al. Hypocholesterolemia in cyanotic congenital heart disease in children. Pediatr Cardiol Cardiac Surg (official J of Japanese Society of Pediatric Cardiology and Cardiac Surgery 2006;22:432.

Cite this article as: Niwa K. Metabolic syndrome and coronary artery disease in adults with congenital heart disease. Cardiovasc Diagn Ther 2021;11(2):563-576. doi: 10.21037/cdt20-781
100.Lui GK, Fernandes S, McElhinney DB. Management of cardiovascular risk factors in adults with congenital heart disease. J Am Heart Assoc 2014:e001076.

101.Joffres M, Shields M, Tremblay MS, et al. Dyslipidemia prevalence, treatment, control, and awareness in the Canadian health measures survey. Can J Public Health 2013;104:e252-7.

102.Martínez-Quintana E, Rodríguez-González F. Lipoprotein(a) concentrations in adult congenital heart disease patients. Congenit Heart Dis 2014;9:63-8.

103. Ohuchi H, Miyamoto Y, Yamamoto M, et al. High prevalence of abnormal glucose metabolism in young adult patients with complex congenital heart disease. Am Heart J 2009;158:30-9.

104.Dellborg M, Bjork A, Fard MNP, et al. High mortality and morbidity among adults with congenital heart disease and type 2 diabetes. Scand Cardiovasc J 2015;49:344-50.

105. Ohuchi H, Yasuda K, Ono S, et al. Low fasting plasma glucose level predicts morbidity and mortality in symptomatic adults with congenital heart disease. Int J Cardiol 2014;174:306-12. 